MKG-Chirurg 2021 · 14:338-340

https://doi.org/10.1007/s12285-021-00328-z

Angenommen: 24. August 2021

Online publiziert: 5. Oktober 2021

(c) Springer Medizin Verlag GmbH, ein Teil von Springer Nature 2021

\section{Redaktion}

Jörg-Ulf Wiegner, Saalfeld/Saale
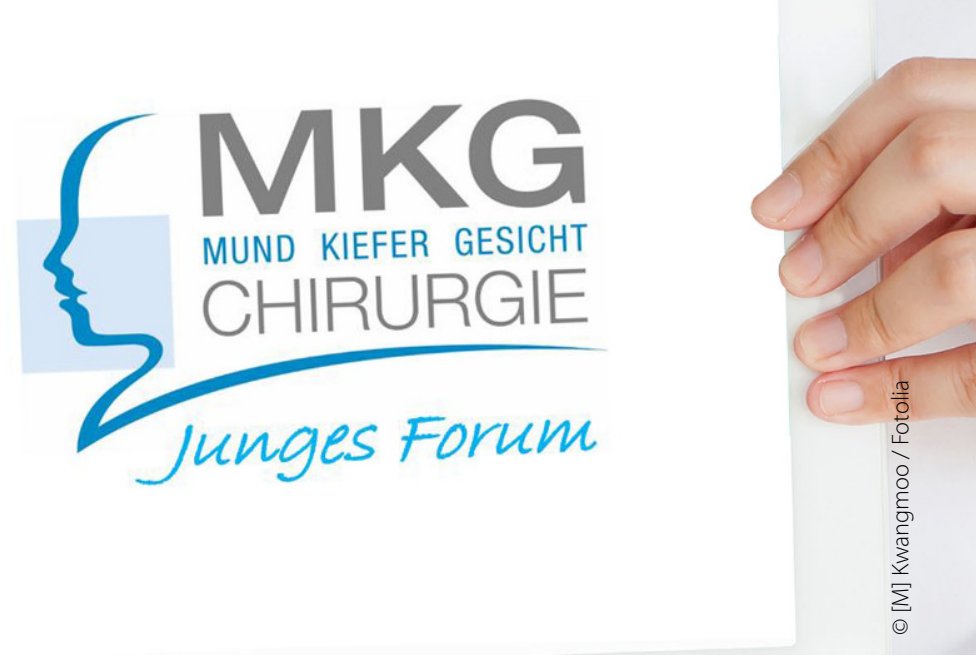

\title{
Junges Forum der DGMKG feiert 10-jähriges Jubiläum
}

\section{Lukas B. Seifert}

Klinik für Mund-, Kiefer- und Plastische Gesichtschirurgie, Universitätsklinikum der Goethe-Universität Frankfurt am Main, Frankfurt am Main, Deutschland
Das Junge Forum der Deutschen Gesellschaft für Mund-, Kiefer- und Gesichtschirurgie (DGMKG) feiert im Jahr 2021 sein 10-jähriges Jubiläum! Im Interview mit Prof. Dr. Dr. Ehrenfeld blicken wir auf die bisherige Geschichte und die Errungenschaften sowie die zukünftig geplanten Projekte des Jungen Forums.

\section{Definition und Ziele}

Das Junge Forum der DGMKG versteht sich als Interessensvertretung von WeiterbildungsassistentInnen im Fach Mund-, Kiefer- und Gesichtschirurgie und jungen FachärztInnen des Fachgebiets in der Weiterbildung zu der Zusatzbezeichnung „Plastische Operationen“.

Ziel des Jungen Forums ist es, das Fachgebiet Mund-, Kiefer- und Gesichtschirurgie mit besonderem Fokus auf die fachliche und berufspolitische Interessensvertretung der jungen KollegInnen, Weiterbildungsmöglichkeiten, Forschung und die Vereinbarkeit von Familie und Beruf attraktiv zu gestalten und weiter zu opti- mieren. Zusätzlich versteht sich das Junge Forum als integratives Netzwerk zum Austausch von Informationen über klinische, wissenschaftliche, didaktische und soziale Aspekte der Weiterbildung. Ein weiteres Ziel ist es, die jungen Kolleglnnen für eine Mitgliedschaft und aktive Mitarbeit im Jungen Forum und in der DGMKG zu gewinnen, um die Stellung der Assistentlnnen innerhalb der DGMKG weiter zu stärken.

\section{Gründung bis 2015}

Das Junge Forum der DGMKG wurde im Jahr 2011 durch die große Unterstützung von Prof. Dr. Dr. Uwe Eckelt (damaliger wissenschaftlicher Leiter der Akademie der DGMKG) und dem damaligen wissenschaftlichen Beirat (Vorgänger des Arbeitskreises Wissenschaft, AkWi) der DGMKG ins Leben gerufen. Ein Assistententreffen fand erstmals am 18. Juni 2011 im Rahmen des Jahreskongresses in Bamberg statt. Erklärtes Ziel war es, „ähnlich dem Jungen Forum der Deutschen Gesellschaft für Orthopädie und Unfallchirurgie eine 


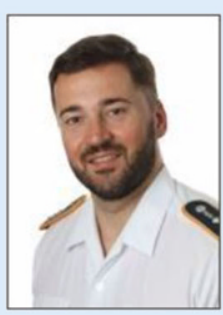

Andreas Pabst Koblenz

Sprecher

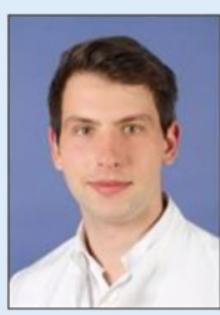

Alexander Zeller Hannover

stellv. Sprecher

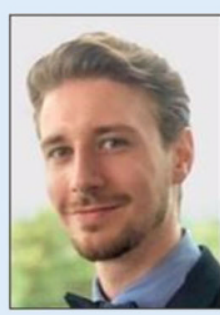

Lukas Seifert

Frankfurt

Lehre

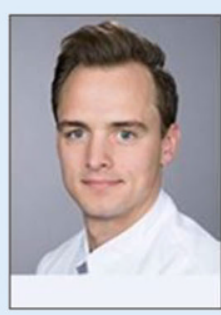

Daniel Thiem Mainz

Kongresse \& Fortbildungen

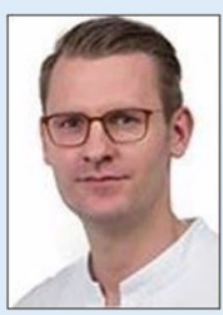

Fabian Beiglböck Basel

Kongresse \& Fortbildungen

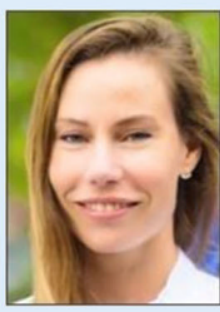

Juliane Kröplin Schwerin

Fachgesellschaften \& DGCH

Perspektivforum Junge Chirurgie

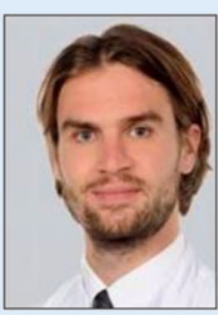

Alexander Bartella Leipzig

Wissenschaft

Abb. 1 A Mitglieder des Jungen Forums der DGMKG der Jahre 2019-2021

Plattform zum gegenseitigen Austausch und eine professionelle, wissenschaftliche und politische Interessensvertretung der WeiterbildungsassistentInnen im Fachgebiet Mund-, Kiefer- und Gesichtschirurgie zu etablieren", so Prof. Dr. Dr. Michael Ehrenfeld, heutiger wissenschaftlicher Leiter der Akademie der DGMKG, der zum damaligen Zeitpunkt intensiv mit in den Gründungsprozess involviert war. Bereits auf dem Jahreskongress der DGMKG 2013 in Essen konnte mit Unterstützung der Akademie der DGMKG der erste Assistententag des Jungen Forums etabliert werden. Geleitet wurde die Veranstaltung von den ersten beiden Assistentensprechern des Jungen Forums, Dr. Dr. Majeed Rana (Düsseldorf, jetzt Prof. Dr. Dr.) und Dr. Dr. Sebastian Baumann (München), die am 30. Mai 2012 ihre Aufgaben im Jungen Forum übernommen hatten. Als eine der ersten und wichtigsten Errungenschaft konnte dank der Unterstützung von Prof. Dr. Dr. Ehrenfeld und des damaligen Vorstandes der DGMKG der jährliche Mitgliedsbeitrag für AssistenzärztInnen in der DGMKG auf $50 €$ gesenkt werden. „Dies habe den Einstieg für ein Engagement innerhalb der Fachgesellschaft für AssistenzärztInnen deutlich erleichtert", berichtet Prof. Dr. Dr. Ehrenfeld. Als weiterer Meilenstein konnten auf dem ersten Assistententag die wichtigsten Ziele des Jungen Forums in Form von 4 Säulen konzentriert werden, hierunter zählen bis heute die Aus- und Weiterbildung, die Vereinbarkeit von Beruf und Familie, die Nachwuchsförderung in der DGMKG so- wie die Integration in Klinik, Wissenschaft und Lehre. Auch wurde zur besseren Vernetzung, zum gegenseitigen Informationsaustausch und zur Unterstützung der AssistenzärztInnen bereits früh eine Facebook-Gruppe (mittlerweile nicht mehr aktiv) gegründet und damit den aktuellen Bestrebungen des Jungen Forums zur Implementierung seiner Aktivitäten auf Social-Media-Plattformen vorgegriffen.

\section{5 bis 2019}

Am 10. Juni 2015 wurden auf dem Assistententag innerhalb des Jahreskongresses der DGMKG in Stuttgart neue Mitglieder des Jungen Forum gewählt. Das Amt des Sprechers und des stellvertretenden Sprechers übernahmen Dr. Dr. Eik Schiegnitz (Mainz, jetzt Priv.-Doz. Dr. Dr.) und Dr. Dr. Maximilian Wagner (Zürich). Außerdem konnten Dr. Dr. Ashkan Rashad (Hamburg) und Dr. Dr. Alexander-N. Zeller (Hannover) als Leiter der Arbeitsgruppen „Planung des Jahreskongresses" und "Nachwuchsgewinnung" gewonnen werden. Unter der Leitung von Dr. Dr. Eik Schiegnitz (jetzt Priv.-Doz. Dr. Dr.) konnte direkt an die wertvolle Arbeit der Vorgänger angeknüpft und diese weiter ausgebaut werden. Auf dem 4. Assistententag innerhalb des Jahreskongresses der DGMKG in Hamburg 2016 wurden, neben vielen interessanten Vorträgen zu den Themenbereichen Onkologie, Traumatologie und Rekonstruktion, auch zwei praktische und sehr gut besuchte ImplantologieWorkshops unter der Leitung von Prof. Dr. Dr. Al-Nawas für die AssistenzärztInnen an- geboten. Als weiteres Angebot wurde im Jahr 2016 zudem auf dem jährlichen Internationalen Symposium für Mund-, Kiefer und Gesichtschirurgen, Oralchirurgen, Zahnärzte und Kieferorthopäden in St. Anton am Arlberg (Österreich) erstmals ein Assistententag des Jungen Forums etabliert. Seitdem findet der Assistententag in St. Anton am Arlberg jährlich statt und bietet neben vielen interessanten Themen, Workshops und einer Industrieausstellung auch die Möglichkeit zum gegenseitigen Austausch und Kennenlernen in sehr netter Atmosphäre.

Im Rahmen des Jahreskongresses der DGMKG in Frankfurt 2019 konnten beim 7. Assistententag auch erstmals wissenschaftliche Akzente durch das Junge Forum gesetzt werden. Dr. Dr. Andreas Pabst (Koblenz) stellte zusammen mit Dr. Dr. Alexander-N. Zeller (Hannover) die Ergebnisse der ersten bundesweiten Erhebung zur aktuellen Weiterbildungssituation der WeiterbildungsassistentInnen in der Mund-, Kiefer- und Gesichtschirurgie vor, die in der Folge international publiziert wurden. Die Daten hierzu wurden im Rahmen des Assistententages in Köln/Bonn 2 Jahre zuvor erstmals erhoben. Dr. Dr. Eik Schiegnitz (jetzt Priv.Doz. Dr. Dr.), der mittlerweile als Oberarzt in der Klinik für Mund-, Kiefer- und Gesichtschirurgie - Plastische Operation der Universitätsmedizin Mainz tätig ist, legte sein Amt als Sprecher des Jungen Forums nach 4-jährigem Engagement nieder und übergab den Staffelstab im Rahmen der offiziellen Neuwahlen an Dr. Dr. Andre- 
as Pabst und Dr. Dr. Alexander-N. Zeller (stellv. Sprecher; - Abb. 1).

\section{9 bis 2021}

An die sehr erfolgreiche Vorarbeit konnte das neu gewählte Junge Forum unter der Leitung von Dr. Dr. Pabst und Dr. Dr. Zeller direkt anknüpfen und diese noch weiter ausbauen. Neben den schon bestehenden Weiterbildungsangeboten im Rahmen des Jahreskongresses der DGMKG und des Internationalen Symposiums für Mund-, Kiefer- und Gesichtschirurgen, Oralchirurgen, Zahnärzte und Kieferorthopäden in St. Anton am Arlberg (Österreich) konnte durch Unterstützung von Prof. Dr. Dr. Frank Hölzle (Aachen) am 16. November erstmals ein kostenfreies Fortbildungstreffen des Jungen Forums zum Thema "Das mikrovaskuläre Fibulatransplantat" im Rahmen der 50. Jahrestagung des Deutsch-Österreichisch-Schweizerischen Arbeitskreises für Tumoren im Kiefer- und Gesichtsbereich (DÖSAK) etabliert werden. Neben vielen hochkarätigen Vorträgen zur computerassistierten Rekonstruktionsplanung wurde es den Teilnehmenden ermöglicht, auch selbst ein Fibulatransplantat inklusive Schnittschablonen unter Anleitung des Aachener Teams um Prof. Dr. Dr. Hölzle digital zu planen. Das Jahr 2020 und die beginnende COVID-19-Pandemie stellten auch das Junge Forum vor große Herausforderungen. Der geplante Jahreskongress der DGMKG in Hamburg musste kurzfristig abgesagt werden, womit auch der 8. Assistententag des Jungen Forums entfiel. Die verschärften Reise- und Kontaktbeschränkungen boten jedoch auch die Chance, neue Formate in der Lehre und Weiterbildung zu erproben. So wurde als Ersatz für den ausgefallenen Assistententag eine Onlinefortbildungsreihe etabliert, die ihr Debüt am 07.09.2020 zum Thema "Navigationsgesteuerte und PSIbasierte Rekonstruktion komplexer Mittelgesichtsfrakturen" (Prof. Dr. Dr. Frank Wilde, Ulm) feierte. Der Umstieg auf eine monatliche Onlinefortbildung stellte sich rückblickend als Glücksgriff heraus, da jeden Monat hochkarätige Referenten zu interessanten und abwechslungsreichen Themen vom „avaskulärem Beckenkammtransplantat" über die "Behandlung von Lippen-Kiefer-Gaumen-Spalten“ bis hin zu den "Möglichkeiten der plastischen und ästhetischen Gesichtschirurgie" referierten und referieren und damit die stetig wachsende Zuhörendenzahl, zu denen nicht nur AssistenzärztInnen zählen, begeistern konnten und können.

Auch der letzte Assistententag am 12. April 2021 wurde aufgrund der zu dieser Zeit hohen COVID-19-Inzidenzraten in digitaler Form im Rahmen des 139. Deutschen Chirurgen Kongresses abgehalten, was jedoch zu keinen Qualitätseinbußen der Beiträge führte. Mit den Themen Möglichkeiten digitaler Lösungen in der Alveolarkammaugmentation (Priv.-Doz. Dr. Dr. Eik Schiegnitz, Mainz), Pitfalls der Dysgnathiechirurgie (Prof. Dr. Dr. Bernd Lethaus, Leipzig) bis zum Einsatz gestielter Fernlappentransplantate (Prof. Dr. Dr. Marco Kesting, Erlangen) und der plastischästhetischen Gesichtschirurgie (Dr. Dr. Wolfgang Funk, München) konnte wieder ein abwechslungsreiches Programm mit renommierten Referenten für die Hörerschaft zusammengestellt werden. Einziger Wehrmutstropfen war, dass ein gemütliches Zusammenkommen am Abend nicht, wie in gewohnter Weise, möglich war. Nach 2-jährigem Vorsitz und Engagement mit vielen Höhen und wenigen Tiefen (COVID-19-Pandemie) übergaben auch Dr. Dr. Andreas Pabst und Dr. Dr. Alexander-N. Zeller das Zepter an die neu gewählten Sprecher des Jungen Forums, Dr. Dr. Daniel Thiem (Mainz, Sprecher) und Dr. Dr. Lukas Seifert (Frankfurt, stellv. Sprecher), und es wurde zum ersten Mal eine Geschäftsordnung für das Junge Forum konzertiert.

\section{Fazit und Ausblick}

In seiner mittlerweile 10-jährigen Existenz hat sich das Junge Forum der DGMKG von einem kleinen Assistententreffen auf dem Jahreskongress der DGMKG in Bamberg 2011 zu einer echten Interessensvertretung aller WeiterbildungsassistentInnen im Fachgebiet Mund-, Kiefer- und Gesichtschirurgie entwickelt und auf zahlreichen Jahreskongressen der DGMKG, des DÖSAK, im Rahmen von Onlinefortbildungen oder, wie zuletzt, auf dem Praxisführungsseminar der DGMKG Weiterbildungsveranstaltungen und Assistentenabende zum gemeinsamen Kennenlernen und
Austausch in lockerer Atmosphäre speziell für uns AssistentInnen organisiert. Dies wäre ohne das unermüdliche und oft auch sehr zeitintensive Engagement der bisherigen Sprecher und Mitglieder des Jungen Forums sowie durch die Unterstützung der DGMKG, der Geschäftsstelle der DGMKG, der Akademie der DGMKG, des AkWi und des Berufsverbands nicht möglich gewesen. Die hinterlassenen großen Fußstapfen gilt es zukünftig mit weiteren Veranstaltungen und Fortbildungsangeboten, Umfragen und wissenschaftlichen Aktivitäten sowie AssistentInnenabenden zum Kennenlernen und Austauschen zu füllen. Der Autor dieses Beitrags bedankt sich deshalb herzlich bei allen VorgängerInnen innerhalb des Jungen Forums für ihr bisheriges Engagement und die vielen bislang erzielten Errungenschaften.

\section{Korrespondenzadresse}

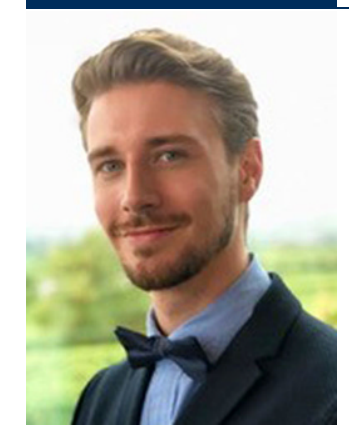

Dr. med. Dr. med. dent. Lukas B. Seifert Klinik für Mund-, Kiefer- und Plastische Gesichtschirurgie, Universitätsklinikum der Goethe-Universität Frankfurt am Main Theodor-Stern-Kai 7, Haus 23 B, UG, 60590 Frankfurt am Main, Deutschland lukasbenedikt.seifert@kgu.de 